\title{
Perfiles docentes y excelencia: un estudio en la Universidad Técnica Particular de Loja, Ecuador
}

\section{Teacher profiles and excellence: $A$ study at the Universidad Técnica Particular of Loja, Ecuador}

Ruth Marlene Aguilar Feijoo

Universidad Técnica Particular de Loja, UTPL (Ecuador)

Ma José Bautista Cerro-Ruiz

Universidad Nacional de Educación Distancia, UNED (España)

\section{Resumen}

El docente universitario constituye un pilar fundamental en la mejora de la calidad de la educación superior, la formación de las nuevas generaciones y la innovación educativa. Su rol no es único y experimenta modificaciones a medida que cambia el enfoque o perspectiva desde el cual se concibe y gestiona el proceso de aprendizaje. Por lo tanto, requiere de un perfil competencial que le permita asumir con éxito los retos que el nuevo paradigma educativo y la sociedad de la información reclaman.

La finalidad principal del presente trabajo de investigación es identificar las competencias que poseen en la actualidad los profesores bimodales de la Universidad Técnica Particular de Loja (Ecuador), a fin de promover el desarrollo de aquellas que no han alcanzado un nivel adecuado. Asumimos la premisa de que estos profesionales deben actuar en un entorno cada vez más complejo, plural, con un acelerado desarrollo tecnológico y demandas formativas diversificadas. Con este propósito se diseñó un cuestionario de autoevaluación que se aplicó a profesores universitarios bimodales de esta Universidad. A partir de los resultados empíricos obtenidos se establecieron tres perfiles competenciales (alto, medio y bajo) e identificaron las competencias específicas y transversales en las que los profesores bimodales tienen un mayor y menor dominio para, desde este conocimiento, determinar sus necesidades formativas. 
Palabras clave: educación superior, competencias docentes, perfil profesional, educación a distancia.

\begin{abstract}
University professors constitute a fundamental pillar in improving the quality of higher education, the training of new generations of students, and in enhancing educational innovation. Their role is often dynamic as it involves making certain modifications. For example, when there is a change of focus or perspective in the way the learning process is conceived or managed. For this reason, university teaches require a competence-based profile that will enable them to successfully take on the challenges arising from the educational paradigm, or those stemming from the needs of our current information society.

The core objective of this current research is to identify the competences that bimodal professors possess at the Universidad Técnica Particular de Loja (Ecuador). By so doing, we can identify the training and development needs of those teaching professionals that have not reached an adequate level of knowledge. This paper is based on the key premise that university professionals have to function in an environment that is becoming more and more complex and multi-focused. Indeed, it is primarily characterized by accelerated technological developments and training needs of a diversified nature. Within this context, we designed a self-evaluation quiz that was applied to bi-modal university professors. Based on the empirical results that were obtained, we were able to establish three competency-based profiles: (high, medium and low), and identify the specific and transversal competences in which the bi-modal professors showed greater or less dominium, i.e. to determine their specific training needs.
\end{abstract}

Keywords: Higher Education, teacher competencies, profiles, distance education.

\title{
LA UNIVERSIDAD Y EL PROFESOR BIMODAL
}

Vivimos una auténtica revolución tecnológica con un fuerte impacto sobre la educación universitaria, movimiento que está transformando las metodologías, estrategias didácticas y prácticas docentes, generando nuevas responsabilidades y retos a las instituciones de educación superior. En este contexto, el docente universitario debe desarrollar competencias que le permitan asumir nuevos roles y construir nuevos y creativos escenarios e itinerarios de aprendizaje. En este entorno 
emergente el docente universitario se perfila como "un diseñador de métodos de aprendizaje, un suscitador de situaciones o ambientes de aprendizaje, capaz de trabajar en equipo con sus alumnos y con otros profesores" (Tünnermann, 2003, p. 158); o como "pedagogoinvestigador" (Recio, 2010, p. 27) artífice en la formación de las nuevas generaciones, aunque "no seamos los únicos" en esta tarea, como lo advierte Zabalza (2005, p. 103).

Frente a esta realidad surge la pregunta ¿cuál debe ser el perfil del profesor universitario para asumir con éxito su trabajo? y, de ahí la preocupación: ¿Los profesores bimodales que ejercen la docencia universitaria en la modalidad presencial y a distancia, reúnen las competencias necesarias para ubicar en el centro del proceso a los estudiantes y conducirles a "aprender a aprender", "aprender a desaprender", "aprender a emprender" y "aprender a arriesgarse"? (Tünnermann, 2011, p. 31). Ardua tarea, en la que se necesitan cambios significativos en los docentes y en las universidades, "con grandes dosis de innovación, ilusión y formación inicial y permanente”, señalan Bozu y Canto (2009, p. 90).

Las diferentes experiencias formativas que se han desarrollado tanto en Europa como en Latinoamérica, nos confirman la pertinencia de un modelo de universidad para el s. XXI que combine los mejores aspectos de la presencialidad y la educación a distancia. Su desarrollo se ha visto facilitado por las innumerables potencialidades que ofrecen las TIC para crear entornos de aprendizaje que puedan responder a las necesidades formativas de los estudiantes. Una ventaja adicional la encontramos en que se facilita el acceso a la educación superior de un amplio sector de la población que de otra manera quedaría, por distintos motivos, excluido de la misma.

La enseñanza bimodal es una de las alternativas que posibilita aprovechar los beneficios de la enseñanza presencial y a distancia en un modelo que permite cubrir y combinar adecuadamente ambos tipos de enseñanza. Cada vez es mayor el número de universidades que inician programas formativos con entornos y modalidades educativas diversas (presencialy a distancia; presencial y semipresencial; distancia tradicional y virtual), debiendo los profesores asumir nuevos roles y 
funciones para los que requieren nuevas competencias. Coincidimos con Yábar, et al. (2011, p. 11), cuando afirman que "el modelo bimodal ha permitido dar respuesta a diferentes situaciones que con un formato totalmente presencial o totalmente a distancia no se podría cumplir". Se abren posibilidades extraordinarias debido a que "la educación bimodal adopta las ventajas ofrecidas por las TIC para superar las barreras del espacio y el tiempo disminuyendo las limitaciones de la presencialidad" (Bustos, 2005, p. 85). Es un modelo que combina lo positivo de la formación presencial con lo mejor de la educación a distancia. Desarrolla procesos formativos flexibles en los que se pone especial énfasis en la interacción, participación y autonomía del estudiante. Todo ello con la finalidad de conseguir aprendizajes significativos a través de un proceso más dinámico y motivador. El uso de determinados dispositivos y software, así como la creación y difusión de contenidos tienen una importancia trascendental ya que "inciden directa o indirectamente en el desarrollo de las personas y de los pueblos, hasta tal punto que para muchos el acceso a las tecnologías computacionales constituye ya un derecho humano" (García-Gutiérrez, 2013, p. 132).

\section{LAS COMPETENCIAS DEL PROFESOR UNIVERSITARIO}

Este modelo, de características determinadas, precisa también de unos docentes capaces de actuar en él con plenas garantías de éxito. Nos encontramos ante la necesidad de identificar las competencias que requiere el profesor universitario bimodal y establecer un perfil actualizado (Aguilar Feijoo, 2008). Este docente debe ser capaz de asumir los retos del nuevo modelo educativo en el que se conjugan los avances tecnológicos, las nuevas metodologías y enfoques pedagógicos. Para afrontar los cambios ya acontecidos y los venideros hay que ser conscientes de que el profesorado es uno de los pilares fundamentales y elemento clave en la calidad de la docencia y, por extensión, de la institucional. Por lo tanto, no se puede desarrollar ninguna innovación, cambio o reforma educativa, sin contar con el compromiso decidido de los docentes (Mas Torelló y Pozos, 2012). 
También es importante no perder de vista que en este nuevo enfoque centrado en la persona que aprende, el docente universitario es el mediador, el facilitador del aprendizaje. Podemos considerar que, básicamente, sus ámbitos de actuación son tres: primero, organizar/ diseñar entornos y experiencias de aprendizaje, segundo, seleccionar y aplicar estrategias, técnicas y recursos innovadores que motiven, activen la curiosidad intelectual y dinamicen el aprendizaje y, en tercer lugar, prestar apoyo a los estudiantes a través de una acción tutorial con énfasis en la autonomía (ICE-U de Zaragoza, 2004) para orientarretroalimentar el trabajo y el aprendizaje de los estudiantes; es decir, lo que Zabalza (2008, p. 10) llama un "aprendizaje acompañado”.

Antes de identificar cuáles son las competencias que requiere el profesor universitario para nosotros fue necesario precisar ¿qué se entiende por competencia? Esta tarea implicó una revisión minuciosa de autores que han investigado en este campo como: Zabalza (2003, 2005); Saravia (2004); García Aretio (2007, 2014); Mas Torrelló (2009, 2011); Legault (2012); Reyero (2014); Adell (2014), entre otros. Además de las reformas en la legislación ecuatoriana, país dónde realizamos esta investigación (Ley Orgánica de Educación Superior, 2010; RRA, 2013). También se han revisado propuestas de perfiles del docente universitario: Valcárcel (2003); Corominas (2006); Tobón (2006); Villa y Poblete (2008); UNESCO (2008); Cabero (2009); Bozu y Canto (2009); Pavié (2012), lo que proporcionó una visión amplia y los fundamentos necesarios sobre las competencias y el perfil ideal del profesor universitario, instrumento de partida para esta investigación.

De todas las definiciones y reflexiones revisadas, la propuesta de Tardif (2006), fue la que nos resultó más completa y actual al concebir la competencia como un 'saber actuar complejo', destacando el carácter integrador, la combinación y movilización de recursos de naturaleza variada (internos y externos) para resolver diversas situaciones del entorno. Desde este enfoque definimos a las competencias docentes como la integración sinérgica de una serie de recursos que el profesor universitario moviliza e integra de manera creativa, reflexiva y crítica, para desempeñar una docencia proactiva, contribuyendo con responsabilidad al desarrollo de las personas y del entorno social. 


\section{METODOLOGÍA}

Lainvestigación objeto deesteestudiose desarrolló en la Universidad Técnica Particular de Loja (UTPL), institución cofinanciada por el Estado ecuatoriano, con más de 40 años de experiencia, que imparte educación universitaria bimodal constituyéndose en una puerta hacia el conocimiento y la profesionalización universitaria. Brinda a 32.000 estudiantes la oportunidad de estudiar 23 titulaciones de grado y varios postgrados, de acuerdo a la disponibilidad de tiempo de los estudiantes, pudiendo elegir una u otra modalidad o las dos a la vez.

Esta investigación comprende tres fases: construcción del perfil ideal del profesor universitario bimodal, elaboración y validación del cuestionario de autoevaluación de competencias docentes y aplicación del cuestionario a los profesores bimodales. Este artículo se centra únicamente en los resultados de esta última fase.

El objetivo fundamental de la misma fue determinar las competencias transversales y específicas que poseen en la actualidad los profesores bimodales de la UTPL y el nivel de dominio de cada una de ellas. A partir de esta información se pretendía promover el desarrollo de aquellas que no han alcanzado un nivel adecuado para asumir en condiciones óptimas las responsabilidades de docencia, investigación, vinculación con la sociedad y gestión, en un entorno cada vez más complejo, con un acelerado desarrollo tecnológico y demandas formativas diversificadas.

\section{Población y muestra}

En la presente investigación se decidió trabajar con una muestra no probabilística, intencional, de acuerdo a dos criterios. El primero, por razones obvias, que fueran profesores bimodales, es decir, que desempeñaran su docencia en las modalidades presencial y a distancia. En segundo lugar, que trabajaran a tiempo completo, debido a que son estos profesionales quienes tienen la responsabilidad de todas las funciones propias de un docente universitario: investigación, docencia, vinculación con la sociedad (extensión) y gestión institucional. 
Con las dos características señaladas: trabajar a tiempo completo y enseñar en las dos modalidades se llegó a identificar a 424 profesores. Teniendo en cuenta las bondades, pero también las limitaciones de una muestra intencional, se decidió realizar la investigación con todo el grupo, a pesar de ser una cantidad considerable para un estudio de este tipo. Esta decisión, permite una mayor seguridad en los resultados obtenidos y, una mayor confianza para su generalización.

Finalmente y tras algunos ajustes necesarios, el grupo objetivo que participó en la presente investigación, y al que se le aplicó el cuestionario de autoevaluación, estuvo conformado por 403 profesores bimodales; de los cuales $58 \%$ eran mujeres y $42 \%$ varones. En relación a la edad es un grupo mayoritariamente joven, el (53\%) se ubican entre los 26 y 35 años y con una experiencia como profesor bimodal en la institución entre 4 y 9 años.

\section{Técnicas y procedimiento seguidos}

Con la finalidad de obtener la información necesaria se diseñó un cuestionario ad hoc, estructurado en dos partes: la primera, constituida por un conjunto de preguntas destinadas a recoger información personal, profesional-académica y laboral de los profesores bimodales. La segunda corresponde al cuestionario de autoevaluación de las competencias docentes.

Para determinar las competencias específicas y transversales que poseen los profesores bimodales y el nivel de dominio alcanzado en cada una de ellas, el instrumento de partida fue el cuestionario de autoevaluación que consta de 114 ítems (indicadores), de los cuales 88 eran bimodales (escalas separadas para modalidad presencial y a distancia), y 26 (competencias transversales) con una sola escala. En todos los casos la escala de respuesta fue de 5 categorías, que indicaban el nivel de dominio del docente (desde $\mathrm{O}=$ No posee el indicador, hasta 4 = Excelente). Para simplificar la escala, se calcularon las correlaciones de Spearman en los 88 ítems bimodales, como forma de determinar la semejanza de los resultados obtenidos en ambas modalidades.

De este modo, se obtuvo una nueva escala con una única respuesta para cada uno delos 114 indicadores originales. Seanalizóla consistencia 
interna del cuestionario, utilizando para ello el índice alfa de Cronbach, obteniéndose valores de 0.88 - 0.99 en la prueba piloto y de $0.92-$ 0.97 en la aplicación final. Los resultados obtenidos en el análisis de la consistencia interna del instrumento mostraron una elevada relación entre los ítems y las competencias, por lo que los resultados para cada una de estas pueden ser resumidos eficientemente utilizando la media de las puntuaciones correspondientes.

Así pues, para determinar el nivel de dominio de los profesores en cada una de las 16 competencias específicas y las 4 transversales, se resumieron las puntuaciones obtenidas en los distintos ítems de cada competencia, utilizando para ello la puntuación media en dichos ítems. A continuación se recodificaron los valores medios obtenidos en una escala de cinco categorías de dominio, idéntica a la empleada originalmente en el instrumento, quedando como se muestra en la tabla 1.

Tabla 1. Categorías de dominio en función de la puntuación media

\begin{tabular}{|c|c|}
\hline Puntuación media & Categorías de dominio \\
\hline$<1$ & o (No posee) \\
\hline $1,1-1,9$ & 1 (Básico) \\
\hline $2-2,9-$ & 2 (Intermedio) \\
\hline $3-3,9$ & 3 (Bueno) \\
\hline$>4$ & 4 (Excelente) \\
\hline
\end{tabular}

\section{RESULTADOS}

\section{Competencias específicas y transversales que poseen los profesores bimodales}

A continuación se presentan los resultados en cuanto al nivel de dominio de los profesores en cada una de las 16 competencias específicas y las 4 transversales, calculadas según el procedimiento anterior. 
Tabla 2. Nivel de domino de las competencias específicas

\begin{tabular}{|c|c|c|c|c|c|c|c|c|c|c|c|c|}
\hline \multicolumn{13}{|c|}{ COMPETENCIAS ESPECÍFICAS } \\
\hline \multirow{4}{*}{$\begin{array}{l}\text { Compe- } \\
\text { tencias }\end{array}$} & \multicolumn{6}{|c|}{ Limitaciones } & \multicolumn{6}{|c|}{ Fortalezas } \\
\hline & \multicolumn{2}{|c|}{$\mathbf{0}$} & \multicolumn{2}{|c|}{$\mathbf{1}$} & \multicolumn{2}{|r|}{2} & \multicolumn{2}{|r|}{$\mathbf{3}$} & \multicolumn{2}{|r|}{4} & \multirow{2}{*}{\multicolumn{2}{|c|}{ TOTAL }} \\
\hline & \multicolumn{2}{|c|}{$\begin{array}{c}\text { No } \\
\text { posee }\end{array}$} & \multicolumn{2}{|c|}{ Básico } & \multicolumn{2}{|c|}{ Intermedio } & \multicolumn{2}{|c|}{ Bueno } & \multicolumn{2}{|c|}{ Excelente } & & \\
\hline & $\mathbf{f}$ & $\%$ & f & $\%$ & f & $\%$ & f & $\%$ & f & $\%$ & $\mathbf{F}$ & $\%$ \\
\hline \multicolumn{13}{|c|}{ Dimensión: Docencia universitaria } \\
\hline $\begin{array}{l}\text { Plani- } \\
\text { ficar la } \\
\text { acción } \\
\text { docente. }\end{array}$ & $\mathrm{O}$ & 0,0 & 46 & 11,4 & 185 & 46 & 167 & 41,4 & 5 & 1,2 & 403 & 100 \\
\hline $\begin{array}{l}\text { Elaborar } \\
\text { mate- } \\
\text { riales y } \\
\text { recursos } \\
\text { educati- } \\
\text { vos. }\end{array}$ & 61 & 15,1 & 35 & 8,7 & 81 & 20,1 & 165 & 41 & 61 & 15,1 & 403 & 100 \\
\hline $\begin{array}{l}\text { Comu- } \\
\text { nicación } \\
\text { con los } \\
\text { estu- } \\
\text { diantes. }\end{array}$ & $\mathrm{O}$ & 0,0 & 9 & 2,2 & 111 & 27,5 & 247 & 61,3 & 36 & 9 & 403 & 100 \\
\hline $\begin{array}{l}\text { Crear un } \\
\text { ambien- } \\
\text { te de } \\
\text { aprendi- } \\
\text { zaje. }\end{array}$ & $\mathrm{O}$ & 0,0 & 24 & 6,0 & 136 & 33,7 & 215 & 53,3 & 28 & 7 & 403 & 100 \\
\hline $\begin{array}{l}\text { Estrate- } \\
\text { gias que } \\
\text { poten- } \\
\text { cien el } \\
\text { desarro- } \\
\text { llo de } \\
\text { compe- } \\
\text { tencias. }\end{array}$ & 0 & 0,0 & 19 & 4,7 & 162 & 40,2 & 203 & 50,4 & 19 & 4,7 & 403 & 100 \\
\hline
\end{tabular}




\begin{tabular}{|c|c|c|c|c|c|c|c|c|c|c|c|c|}
\hline $\begin{array}{l}\text { Crear } \\
\text { opor- } \\
\text { tunida- } \\
\text { des de } \\
\text { aprendi- } \\
\text { zajes. }\end{array}$ & 1 & 0,2 & 36 & 9 & 164 & 40,7 & 189 & 47 & 13 & 3,1 & 403 & 100 \\
\hline $\begin{array}{l}\text { Funcio- } \\
\text { nes de } \\
\text { media- } \\
\text { ción y } \\
\text { tutoría. }\end{array}$ & $\mathrm{O}$ & 0,0 & 39 & 9,7 & 164 & 40,7 & 191 & 47,4 & 9 & 2,2 & 403 & 100 \\
\hline $\begin{array}{l}\text { Utilizar } \\
\text { las TIC } \\
\text { en la } \\
\text { ense- } \\
\text { ñanza. }\end{array}$ & 3 & 0,7 & 48 & 12 & 173 & 43 & 166 & 41,2 & 13 & 3,1 & 403 & 100 \\
\hline $\begin{array}{l}\text { Eva- } \\
\text { luar el } \\
\text { aprendi- } \\
\text { zaje. }\end{array}$ & 6 & 1,5 & 82 & 20,3 & 197 & 49 & 109 & 27,0 & 9 & 2,2 & 403 & 100 \\
\hline $\begin{array}{l}\text { Evaluar } \\
\text { la acti- } \\
\text { vidad } \\
\text { docente. }\end{array}$ & 2 & 0,5 & 48 & 12 & 168 & 41,5 & 169 & 42 & 16 & 4,0 & 403 & 100 \\
\hline \multicolumn{13}{|c|}{ Dimensión: Investigación } \\
\hline $\begin{array}{l}\text { Dise- } \\
\text { ñar y } \\
\text { evaluar } \\
\text { proyec- } \\
\text { tos de } \\
\text { investi- } \\
\text { gación. }\end{array}$ & 34 & 8,4 & 117 & 29,0 & 151 & 37,5 & 91 & 22,6 & 10 & 2,5 & 403 & 100 \\
\hline $\begin{array}{l}\text { Escri- } \\
\text { bir y } \\
\text { publicar } \\
\text { artículos } \\
\text { científi- } \\
\text { cos. }\end{array}$ & 164 & 40,7 & 144 & 35,7 & 59 & 14,6 & 28 & 7 & 8 & 2,0 & 403 & 100 \\
\hline
\end{tabular}




\begin{tabular}{|c|c|c|c|c|c|c|c|c|c|c|c|c|}
\hline $\begin{array}{l}\text { Innovar } \\
\text { la acti- } \\
\text { vidad } \\
\text { docente. }\end{array}$ & 28 & 7 & 131 & 32,5 & 171 & 42,4 & 66 & 16,4 & 7 & 1,7 & 403 & 100 \\
\hline \multicolumn{13}{|c|}{ Dimensión: Vinculación con la sociedad } \\
\hline $\begin{array}{l}\text { Elaborar } \\
\text { pro- } \\
\text { yectos } \\
\text { para la } \\
\text { solución } \\
\text { de pro- } \\
\text { blemas } \\
\text { de la } \\
\text { comuni- } \\
\text { dad. }\end{array}$ & 39 & 9,7 & 132 & 32,7 & 147 & 36,5 & 80 & 19,9 & 5 & 1,2 & 403 & 100 \\
\hline \multicolumn{13}{|c|}{ Dimensión: Gestión universitaria } \\
\hline $\begin{array}{l}\text { Contri- } \\
\text { buir al } \\
\text { desarro- } \\
\text { llo de la } \\
\text { institu- } \\
\text { ción. }\end{array}$ & 6 & 1,5 & 47 & 11,6 & 128 & 31,8 & 164 & 40,7 & 58 & 14,4 & 403 & 100 \\
\hline $\begin{array}{l}\text { Aplicar } \\
\text { estrate- } \\
\text { gias de } \\
\text { gestión. }\end{array}$ & 29 & 7,2 & 84 & 20,8 & 140 & 34,7 & 124 & 30,8 & 26 & 6,5 & 403 & 100 \\
\hline $\begin{array}{l}\text { Nivel } \\
\text { de do- } \\
\text { minio } \\
\text { de las } \\
\text { compe- } \\
\text { tencias }\end{array}$ & \multicolumn{2}{|c|}{$\begin{array}{c}1 \\
\text { No poseen }\end{array}$} & \multicolumn{2}{|c|}{$\begin{array}{c}\text { o } \\
\text { Básico }\end{array}$} & \multicolumn{2}{|c|}{$\begin{array}{c}8 \\
\text { Intermedio }\end{array}$} & \multicolumn{2}{|c|}{$\begin{array}{c}7 \\
\text { Bueno }\end{array}$} & \multicolumn{2}{|c|}{$\begin{array}{c}\mathrm{o} \\
\text { Excelente }\end{array}$} & \multicolumn{2}{|c|}{16} \\
\hline
\end{tabular}

Fuente: Instrumento de autoevaluación. Elaboración propia

El análisis del logro de las 16 competencias específicas se ha realizado en función de las cuatro dimensiones en las cuales el docente desarrolla sus actividades (docencia universitaria, investigación, vinculación con la colectividad y gestión institucional) y nos referiremos solo a los 
porcentajes que concentran el mayor número de profesores en cada competencia.

a. Docencia universitaria. Se han contemplado 10 competencias, en 3 de las cuales más del 50\% de los profesores bimodales tienen un nivel de dominio bueno. Estas son:

- $\quad$ Comunicación con los estudiantes (61,3\%).

- Crear un ambiente de aprendizaje (53,3\%).

- $\quad$ Proponer estrategias (actividades y recursos) que potencien el desarrollo de las competencias $(50,4 \%)$.

Además, si sumamos los porcentajes de bueno y excelente, encontramos que más del 50\% de los profesores investigados han alcanzado un buen nivel de dominio en otras 3 competencias para la docencia:

- Elaborar la guía didáctica y otros materiales y recursos educativos (56,1\%).

- $\quad$ Contribuir al desarrollo de la institución (55,1\%).

- $\quad$ Crear oportunidades de aprendizaje individual y grupal (50,1\%).

También en este mismo ámbito de la docencia, más de la mitad de los profesores bimodales tienen serias limitaciones en 4 de las 10 competencias específicas. En tres competencias presentan un nivel de dominio intermedio y un nivel básico en una de ellas. Así:

- $\quad$ Evaluar y retroalimentar los resultados del aprendizaje (69,3\%).

- $\quad$ Prepararse y planificar la actividad docente (57,4\%).

- Utilizar pedagógicamente las TIC (55\%).

- Evaluar, e interpretar los resultados de la actividad docente $(53,5 \%)$.

b. Investigación. De las 3 competencias consideradas en el perfil del profesor bimodal, el nivel de dominio alcanzado oscila entre no posee (o) y básico (1). Revisemos los resultados: 
- $\quad$ Escribir y publicar artículos científicos. El 40,7\% de los docentes no posee (o) esta competencia; si a este resultado sumamos el 35,7\% de profesores que tienen esta competencia en el nivel básico (1), obtenemos un porcentaje muy alto $(76,4 \%)$ de profesores que no disponen de los conocimientos, habilidades, actitudes y motivaciones para investigar.

- Innovar y mejorar la actividad docente. El 42,4\% alcanza el nivel intermedio y un $32,5 \%$ el nivel básico, lo que significa que el $74,9 \%$ de los 403 profesores no se siente competente para realizar innovaciones en su labor docente, lo que resulta comprensible, porque si no se involucran en investigación, difícilmente surgirá la innovación.

- Diseñar y evaluar proyectos de investigación. En relación a esta competencia el nivel de dominio de los profesores es intermedio $(37,5 \%)$ y básico (29\%); valores que confirman las limitaciones que la mayoría de los profesores tienen para realizar tareas de investigación.

Estos resultados nos permiten concluir que los profesores bimodales que formaron parte del estudio no poseen un buen dominio de las competencias para desempeñarse con idoneidad en el campo de la investigación e innovación. Así mismo, se puede afirmar que el 36\% de los profesores no están involucrados en actividades de investigación, y, en igual porcentaje, tampoco participa en proyectos de investigación y el $38,7 \%$ no dedica ningún tiempo a la investigación científica, lo que debe plantear la necesidad de fomentar espacios que faciliten la formación y desarrollo en estas competencias.

a. Vinculación con la sociedad. Es la tercera función asignada al profesor universitario por la Ley Orgánica de Educación Superior (2010, art. 13, literal a) y el Reglamento de Carrera y Escalafón Docente (2012, art. 9). Estas actividades vienen a renovar la función clásica de la extensión universitaria. 
En el perfil se incluyó la competencia: Proponer proyectos y emprender acciones y actividades orientadas a la solución de problemas de la comunidad, involucrando a los estudiantes. Los resultados igualmente no son del todo alentadores, porque la mayoría de los profesores bimodales solo han logrado niveles intermedio $(36,5 \%)$ y básico $(32,75 \%)$. Valores que reflejan que un alto porcentaje de profesores $(69,25 \%)$ aún no han alcanzado un nivel adecuado para desempeñarse con idoneidad en este campo, que deberá ser fomentado en diferentes escenarios de esta institución.

b. Función de gestión institucional. Finalmente, la cuarta función a la que los profesores universitarios bimodales asignan gran parte de su jornada de trabajo es a las actividades de gestión institucional. Según su propia percepción el 46,9\% de los docentes investigados dedican entre el $20 \%$ y el $50 \%$ de su tiempo a esta función.

Dos son las competencias que se incluyeron para detectar esta dimensión:

- La primera, orientada a contribuir en el desarrollo y buen funcionamiento de la institución, en la cual el 40,7\% de los profesores alcanzan el nivel 3 (bueno). Sin embargo, hay un importante $31,8 \%$ que solo llegan al nivel intermedio.

- La segunda competencia implica aplicar estrategias de gestión que favorezcan el trabajo colaborativo de los equipos docentes. Es la competencia en la que se advierten grandes limitaciones. Así, el 20,8\% apenas tienen un nivel básico y el 34,7\% intermedio. Podemos entender pues que el 55,5\% de los profesores, a pesar de que tienen la motivación y el compromiso para colaborar en la gestión institucional, no cuentan con el dominio de las estrategias necesarias para gestionar las diferentes tareas encomendadas.

En este estudio no existen porcentajes significativos de profesores que alcancen el nivel de excelencia. Solo en 7 competencias los porcentajes más altos logran el nivel bueno y en la mayoría de ellas (8) los profesores presentan un nivel de dominio intermedio y una no 
la poseen. Lo que nos permite concluir que en 9 de las competencias específicas los profesores bimodales tienen limitaciones.

En relación a las competencias transversales, dos de las cuales corresponden a valores y actitudes personales y las otras dos a interpersonales, los resultados son más homogéneos, identificándose que la mayoría de los profesores poseen tres competencias en el nivel bueno: Comprometerse en la tarea docente (66,7\%), Promover el desarrollo de un espíritu colegiado $(58,3 \%)$ y Capacidad de liderazgo (61,5\%). La competencia Cumplir con integridad las diferentes funciones se encuentra distribuida en porcentajes similares en dos niveles: bueno (48\%) y excelente (47,1\%), información que se puede observar en la siguiente tabla:

Tabla 3. Nivel de dominio de las competencias transversales

\begin{tabular}{|c|c|c|c|c|c|c|c|c|c|c|c|c|}
\hline \multicolumn{13}{|c|}{ COMPETENCIAS TRANSVERSALES } \\
\hline \multirow[t]{2}{*}{ Competencias } & \multicolumn{2}{|c|}{$\begin{array}{c}0 \\
\text { No } \\
\text { posee }\end{array}$} & \multicolumn{2}{|c|}{$\begin{array}{c}1 \\
\text { Básico }\end{array}$} & \multicolumn{2}{|c|}{$\begin{array}{c}2 \\
\text { Intermedio }\end{array}$} & \multicolumn{2}{|c|}{$\begin{array}{c}3 \\
\text { Bueno }\end{array}$} & \multicolumn{2}{|c|}{$\begin{array}{c}4 \\
\text { Excelente }\end{array}$} & \multicolumn{2}{|c|}{ TOTAL } \\
\hline & $\mathbf{f}$ & $\%$ & $\mathbf{f}$ & $\%$ & $\mathbf{f}$ & $\%$ & $\mathbf{f}$ & $\%$ & $\mathbf{f}$ & $\%$ & $\mathbf{f}$ & $\%$ \\
\hline $\begin{array}{l}\text { Cumplir con } \\
\text { integridad } \\
\text { las diferentes } \\
\text { funciones. }\end{array}$ & 0 & 0,0 & 3 & 0,7 & 17 & 4,2 & 193 & 47,9 & 190 & 47,1 & 403 & 100 \\
\hline $\begin{array}{l}\text { Comprometer- } \\
\text { se con respon- } \\
\text { sabilidad en la } \\
\text { docencia. }\end{array}$ & $\mathrm{O}$ & 0,0 & 2 & 0,5 & 19 & 4,7 & 269 & 66,7 & 113 & 28,0 & 403 & 100 \\
\hline $\begin{array}{l}\text { Promover el } \\
\text { desarrollo del } \\
\text { espíritu cole- } \\
\text { giado. }\end{array}$ & 5 & 1,2 & 14 & 3,5 & 90 & 22,3 & 235 & 58,3 & 59 & 14,5 & 403 & 100 \\
\hline $\begin{array}{l}\text { Capacidad de } \\
\text { liderazgo. }\end{array}$ & $\mathrm{O}$ & 0,0 & 16 & 4,0 & 115 & 28,5 & 248 & 61,5 & 24 & 6,0 & 403 & 100 \\
\hline
\end{tabular}

Fuente: Instrumento de autoevaluación. Elaboración propia 


\section{Perfil del docente bimodal de la UTPL}

Trasestosresultados, sehanidentificadotres perfiles competenciales en el grupo investigado. En la figura que se incluye a continuación, se aprecia claramente las 20 competencias y la separación entre los 3 grupos, lo que indica una clara diferencia entre ellos en todas y cada una de las competencias.

Figura 1. Perfil competencial de los profesores bimodales

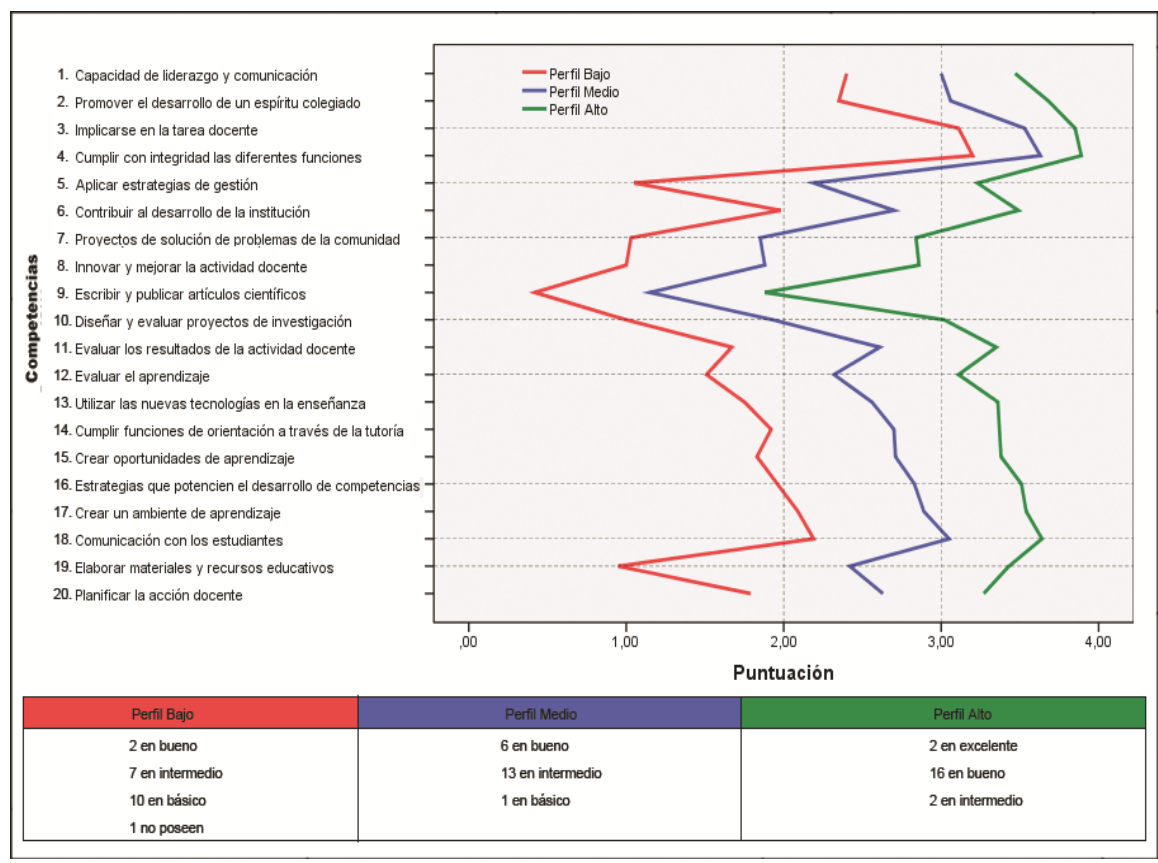

Fuente: Instrumento de autoevaluación

1. Perfil competencial bajo: Este perfil congrega al 6\% (24) profesores bimodales, quienes presentan la mayor parte de las competencias en los niveles más bajos de dominio (no poseen o básico). De las diez competencias que tienen un nivel básico, seis corresponden a la docencia (planificación, elaboración de materiales educativos, crear oportunidades de aprendizaje, utilizar las TIC, evaluar el aprendizaje y los resultados de la actividad docente). De aquellas 
ubicadas en el nivel intermedio, cuatro pertenecen a esta misma función (comunicación con los estudiantes, crear un ambiente de aprendizaje, proponer estrategias para desarrollar competencias y brindar tutoría).

Otro campo en el que detectamos lagunas en los profesores del perfil bajo es en la investigación; de las tres competencias consideradas, una no la poseen (escribir artículos científicos) y dos alcanzan el nivel básico (diseñar y evaluar proyectos de investigación e innovar la actividad docente). Conviene aclarar que si bien estos profesores tienen limitaciones en el dominio de sus competencias, no es un grupo mayoritario.

2. Perfil competencial medio: Este perfil concentra al 60\% (242) de los profesores de la muestra. Estos docentes presentan la mayor parte de las competencias repartidas entre los niveles bueno (6) e intermedio (13) aunque en ninguna de ellas alcanzan un nivel excelente.

Es importantedestacar quelos profesores ubicados en el perfil medio también tienen limitaciones importantes en la docencia universitaria, en la que únicamente dos de las diez competencias tienen un nivel bueno, las ocho restantes alcanzan únicamente el nivel intermedio; situación similar se observa en el campo de la investigación, vinculación con la colectividad y gestión institucional, cuyas competencias logran el nivel intermedio y una el nivel básico.

3. Perfil competencial alto: Este grupo reúne el 34\% (137) de los docentes bimodales. Estos profesionales presentan 16 competencias en el nivel bueno, de las cuales 10 corresponden a la docencia universitaria, 2 a investigación, 2 a gestión institucional y 2 transversales. Es necesario destacar que este grupo posee 2 competencias transversales en el nivel excelente: Cumplir con integridad las diferentes funciones $(47,1 \%)$ y comprometerse con responsabilidad en la tarea docente (28\%). En el nivel intermedio encontramos otras 2: Escribir y publicar artículos (investigación) 
y elaborar proyectos para solucionar problemas de la comunidad (de vinculación con la sociedad).

Los profesores que se ubican en este perfil tampoco alcanzan en todas las competencias el nivel 4 o de excelencia. Sin embargo, poseen un nivel bueno en las diez competencias consideradas para la docencia; lo que significa que este grupo está mejor preparado para la enseñanza universitaria. Aunque, en el ámbito de la investigación y vinculación con la colectividad, tienen limitaciones en dos competencias: escribir y publicar artículos científicos y diseñar proyectos para la solución de problemas de la comunidad, en las que logran un nivel intermedio.

En general, el estudio revela que los docentes bimodales, independientemente del perfil en el que se encuentren ubicados, están mejor preparados para la docencia que para la investigación y la vinculación con la sociedad.

Finalmente, se advierte que los profesores investigados fueron más rigurosos a la hora de autoevaluarse en las competencias específicas y generosos cuando se trata de valores y actitudes (competencias transversales).

\section{DISCUSIÓN}

En este estudio también se relacionaron las características personales y profesionales-académicas con las competencias y el perfil competencial que poseen los profesores bimodales, consiguiéndose datos muy interesantes que precisamos a continuación:

- Los resultados revelan que tanto varones como mujeres están distribuidos en los tres perfiles competenciales; sin embargo, encontramos más mujeres en los tres perfiles: bajo, medio y alto, lo que resulta lógico, porque la población femenina fue notablemente mayor que la de los varones.

- La composición de los tres perfiles por grupo de edad revela que el perfil alto está representado, mayoritariamente, por los profesores de mayor edad. En el perfil medio encontramos una distribución en porcentajes similares entre los diferentes grupos 
de edad, con una pequeña ventaja de los profesores de mayor edad. Finalmente, en relación al perfil bajo, los grupos de profesores de menor edad son mayoría. Son diversos los factores que pueden influir en esta distribución, aunque los datos parecen apuntar a la experiencia como un elemento importante para alcanzar un perfil medio-alto.

- $\quad$ Si relacionamos el perfil competencial con los estudios de Postgrado detectamos que los profesores con título de Especialista, Ph.D. y Maestría se ubican en el perfil alto. En cambio, en el grupo de profesores que no tienen ningún título de Postgrado encontramos el mayor porcentaje de perfiles bajos y el menor porcentaje de altos. Esto nos permite concluir que a mayor título de Postgrado mejor es el perfil competencial y nos invita a pensar en la necesidad de que los profesores de la UTPL se involucren en estudios de Postgrado, ya que esto redundará en beneficio no solo personal, sino también en la propia institución.

- Considerando el tiempo que dedican los profesores a cada una de las tres funciones (docencia, investigación y gestión institucional), observamos que los profesores que dedican, en promedio, más tiempo a la docencia están distribuidos de manera homogénea en los tres perfiles: bajo, medio y alto. Los profesores que asignan en promedio más tiempo a la investigación se ubican en el perfil competencial alto y, finalmente, los que dedican más tiempo a la gestión institucional se ubican en el perfil bajo. Lo que permite concluir que el tiempo dedicado a la investigación repercute en la mejora del perfil competencial. En sentido contrario, cuanto más tiempo dedican los profesores a la gestión, menor es el perfil.

\section{CONCLUSIONES}

En primer lugar, es necesario destacar que los profesores, a través de la autorreflexión y autoevaluación, son capaces de reconocer las fortalezasylimitaciones en su desarrolloprofesional. Esta aproximación al perfil real del profesor bimodal que labora en la UTPL permitió determinar, desde su propia percepción, las fortalezas, limitaciones y 
nodos más problemáticos que tienen en las competencias requeridas para el ejercicio profesional de cada una de las funciones universitarias.

En función de los resultados obtenidos en la investigación se han establecido tres perfiles competenciales para el profesor bimodal: perfil alto que congrega al 34\% de docentes, perfil medio que reúne a la mayoría de los docentes investigados (60\%) y perfil bajo que agrupa al 6\% de docentes.

Y, por tanto, los datos nos permiten obtener un mapa de las principales necesidades y fortalezas de los docentes que deben traducirse en un programa de mejora docente, es decir, en políticas proactivas por parte de la institución educativa interesada en alcanzar la excelencia.

Una de las conclusiones más importantes es que los docentes precisan de apoyo y estímulo, principalmente al inicio de su carrera profesional. Los datos muestran que los docentes con más experiencia tienen mejores perfiles que aquellos más jóvenes. Este hecho es especialmente preocupante ya que los docentes jóvenes conforman la mayor parte del personal docente de nuestra universidad. En paralelo, no podemos pasar por alto que en los perfiles más bajos se sitúan los docentes con menor nivel formativo. Por ello, además del apoyo institucional para la elaboración de programas sistemáticos de buenas prácticas y medidas que promuevan el trabajo en equipo conjuntamente con la realización de proyectos de investigación e innovación docente, entre otras acciones, creemos que es imprescindible que se favorezca y facilite que los profesores de la UTPL se involucren en estudios de Postgrado, sobre todo de Doctorado, dada la estrecha relación entre este y la actividad investigadora.

Para una institución que desee alcanzar la excelencia resulta preocupante tener un 6\% del conjunto de los docentes en el perfil bajo. Este grupo, aunque pequeño, al no reunir las competencias necesarias para desempeñar las funciones universitarias, requiere formación permanente. En este sentido, y dado el porcentaje de profesores/as que se sitúan en el perfil alto, podría ser interesante aprovechar la experiencia y calidad de estos docentes en proyectos de mentoría o similar. 
Por último, nos parecen relevantes las limitaciones evidentes en las tres competencias del ámbito de la investigación: innovar y mejorar la actividad docente, escribir y publicar artículos científicos, diseñar y evaluar proyectos de investigación. Se trata de una situación insostenible, no solo a nivel individual, ya que el profesor que investiga es un profesor que mejora en su práctica docente, sino a nivel institucional. "Todo nuevo paso en el conocimiento debe ser capaz de abrazar todo el conocimiento anterior e ir un paso más allá” (Galán, Ruiz-Corbella y Sánchez, 2014, p. 292). La universidad no puede perder de vista este compromiso que fortalece a la propia institución y, además, supone una mejora en la formación de sus estudiantes.

Laidentificación delos tres perfiles competenciales y el conocimiento del nivel de desarrollo alcanzado por el profesorado bimodal en cada una de las competencias aporta a la institución, información sumamente valiosa y actualizada, que permitirá planificar de manera estructurada y sistemática programas diferenciados, para atender las necesidades específicas de capacitación y formación de los docentes de cada uno de estos perfiles.

Los resultados obtenidos, en relación con las competencias genéricas, nos dejan la inquietud de si en realidad tienen el nivel de dominio que señalaron o faltó una buena dosis de autocrítica a la hora de autoevaluarse. Preocupación que se despejaría, refrendando este estudio con nuevas investigaciones en las que se incluyan el criterio de los estudiantes, de los pares en cada sección departamental y de las autoridades académicas y de investigación.

Finalmente, es importante destacar que la utilización de la autoevaluación, que en principio, podría parecer una debilidad, es una fortaleza, porque es una técnica que permite obtener información estratégica, a través del autoconocimiento que el profesor tiene de sí mismo, se trabaja sobre su inteligencia emocional y autocomprensión. Esto no le quita validez científica al estudio, en la medida que es el profesor quien mejor se conocey, por lo tanto, tiene una autopercepción muy clara de su forma de estar y actuar en la universidad. Además resulta difícil conocer y potenciar el desarrollo de las personas y sus competencias si no se trabaja desde su emocionalidad, autoestima y compromiso con la institución. 


\section{REFERENCIAS BIBLIOGRÁFICAS}

Adell, J. (2014). Las TIC no hacen bueno o malo a un docente. En tiching Blog, 20 de Febrero. Recuperado de http://blog.tiching.com/jordiadell-las-tic-hacen-bueno-o-maloun-docente/.

Aguilar Feijoo, R. (2008). Las competencias básicas del Profesor Bimodal de la UTPL. [Edición especial II Congreso Cread Andes y II Encuentro Virtual Educa. LojaEcuador, abril]. Revista Cognición, 13, Edición Especial. Recuperado de http://www.cognicion.net/ index2. php?option=com content\&do pdf=1\&id=115.

Asamblea Nacional (2010). Ley Orgánica de Educación Superior (LOES). Quito: Registro Oficial $\mathrm{N}^{\circ}$ 298, octubre 2010.

Bustos, A. (2005). Estrategias didácticas para el uso de las TICs en la docencia universitaria presencial. Un Manual para los ciudadanos del Ágora. Valparaíso, Chile: Universidad Católica de Valparaíso. Recuperado de http://eprints.rclis.org/9542/1/ manualedTICS.pdf.

Bozu, Z., y Canto Herrera, P. J. (2009). El profesorado universitario en la sociedad del conocimiento: competencias profesionales docentes. Revista de Formación e Innovación Educativa Universitaria, 2 (2), 8797. Recuperado de http://webs.
uvigo.es/refiedu/Refiedu/Vol2 2/ REFIEDU $2 \quad 2$ 4.pdf.

Cabero, J., Bullón, P., Llorente, M., Machuca, M., Machuca, G., y Marín, V. (2009). Competencias Tecnológicas del profesorado de la Facultad de Odontología de la Universidad de Sevilla. Sevilla: Plublidisa.

Consejo de Educación Superior-CES (2013). Reglamento de Régimen Académico (RRA). Quito: Registro oficial $\mathrm{N}^{\mathrm{o}} 136$, diciembre 2013.

Corominas, E., Tesouro, M., Capell, D., Teixidó, J., Pèlach, J., y Cortada, R. (2006). Percepciones del profesorado ante la incorporación de las competencias genéricas en la formación universitaria. Revista de Educación, 341, 301336. Recuperado de http://www. revistaeducacion.mepsyd.es/re341/ re341 14.pdf.

Galán, A., Ruiz-Corbella, M., y Sánchez Melado, J. C. (2014). Repensar la investigación educativa: las relaciones lineales al paradigma de la complejidad. Revista Española de Pedagogía, 258, 281-298. Recuperado de http:// dialnet.unirioja.es/servlet/ articulo?codigo $=4714477$.

García Aretio, L., Ruiz-Corbella, M., y Domínguez F. D. (2007). De la educación a distancia a la educación virtual. Barcelona: Ariel. 
García Aretio, L. (2014). Bases, mediaciones y futuro de la educación a distancia en la sociedad digital. Madrid: Síntesis.

García-Gutiérrez, J. (2013). Aproximación ética a la competencia digital. Los niveles de uso y sentido en ámbitos educativos virtuales. Revista Teoría de la Educación. Educación y cultura en la sociedad de la información, 14 (3), 121-145. Recuperado de http://campus.usal. es/ revistas trabajo/index.php/ revistatesi/article/view/11354/ 11771.

ICE-U de Zaragoza (2004). El proceso de enseñanza-aprendizaje por competencias. Programa de Mejora e Innovación de la Docencia en el marco de la convergencia al EEES. Zaragoza: ICE-Vicerrectorado Ordenación AcadémicaVicerrectorado de Estudiantes de la Universidad de Zaragoza. Recuperado de http://www.unizar. es/ice/images/stories/profesores/ InnovDocencia2004-2009.pdf.

Legault Avocat, A. (2012). ¿Una enseñanza universitaria basada en competencias? ¿Por qué? ¿Cómo? Revista Electrónica de Desarrollo de Competencias (REDEC), 5 (1). Recuperado de http://redec. utalca.cl/index.php/redec /article/ viewFile/84/97.

Mas Torelló, Ò., y Pozos Pérez, K. (2012). Las competencias pedagógicas y digitales del docente universitario. Un elemento nuclear en la calidad docente e institucional. En VII Congreso Internacional de Docencia Universitaria $e$ Innovación (CIDUI), Barcelona, España. Recuperado de https:// www.academia.edu/2041196/ LAS COMPETENCIAS P E D A G Ó G I C A S Y DIGITALES DEL DOCENTE UNIVERSITARIO Un elemento nuclear en la calidad docente e institucional.

Mas Torelló, Ò. (2011). El profesor universitario: sus competencias y formación. Profesorado. Revista de currículum y formación del profesorado, 15 (3), 195-211. Recuperado de http://www.ugr. es/ recfpro/rev153COL1.pdf.

Pavié, A. (2012). Las competencias profesionales del profesorado de Lengua Castellana $y$ Comunicaciones en Chile: Aportaciones a la formación inicial. [Tesis doctoral]. Universidad de Valladolid, España. Recuperado de http://uvadoc. uva.es/bitstream/10324/2794/1/ TESIS297-130508.pdf.

Poblete Ruiz, M. (2006). Las competencias, instrumento para un cambio de paradigma. En $X$ Simposio de la Sociedad Española de Investigación en Educación Matemática, Huesca, España. Recuperado de http:// dialnet.unirioja.es/servlet/ articulo? codigo $=2264720$. 
Recio Buriticá, Á. (2010). El perfil del educador para el siglo XXI. En Simposio sobre Educación para el siglo XXI,Bogotá, Colombia. Recuperado de https://goo.gl/ mOIDbP.

Reyero, D.(2014). La excelencia docente universitaria. Análisis y propuestas para una mejor evaluación del profesorado universitario. Educación XX1, 17 (2), 125-143. Recuperado de http://revistas. uned.es/index.php/educacionXX1/ article/view/11482/11423.

Saravia, M. A. (2004). Evaluación del profesorado universitario. Un enfoque desde la competencia profesional. [Tesis doctoral]. Universidad de Barcelona, España. Recuperado de http://www.tdx.cat/ handle/10803/2342.

Tardif, J. (2008). Desarrollo de un programa por competencias: De la intención a su implementación. Profesorado. Revista de currículum y formación del profesorado, 12 (3), 36-45. Recuperado de http://www. ugr.es/ recfpro/rev123ART2.pdf.

Tobón, S. (2006). Formación basada en competencias. Pensamiento complejo, diseño curricular $y$ didáctica. Bogotá: ECOE Ediciones. Tünnermann, C. (2003). Desafíos del docente universitario en la educación del siglo XXI. En Tendencias innovadoras en la Educación Superior de América Latina. (157-161). Managua,
Nicaragua: Fundación Enrique Bolaños.

Tünnermann, C. (2011). La universidad del futuro. Managua, Nicaragua: HISPAMER. Recuperado de http://enriquebolanos.org/ CarlosTunnermannBernheim/ La\%20universidad\%2odel\%20 futuro.pdf.

Valcárcel, M. (Coord.). (2003). La preparación del profesorado universitario español para la Convergencia Europea en Educación Superior. Recuperado de http://campus.usal.es/webusal/Novedades/noticias/bolonia/ informe final.pdf.

Villa Sánchez, A., y Poblete Ruiz, M. (2008). Aprendizaje basado en competencias. Una propuesta para la evaluación de las competencias genéricas. Bilbao: Universidad de Deusto.

Yábar, J. M., Barbarà, P. L., y Añaños, E. (2010). Desarrollo de un campus virtual de la comunicación en el marco de una educación bimodal. Barcelona: Centro Virtual Cervantes. Recuperado de http://cvc.cervantes. es/ensenanza/formacion virtual/ campus virtual/yabar.htm.

Zabalza, M. A. (2005). Competencias Docentes. Recuperado de http:// www.moodle.ufba.br/file. $\mathrm{php} / 11739 /$ Equipe EDUMATEC/ Semana1/competencias.pdf.

Zabalza, M. A. (2008). El papel del profesorado en el Espacio 
Europeo de Educación Superior. Modelo Educativo de la Universidad Politécnica de Madrid. (Presentación en power point).
Recuperado de http://es.slideshare. net/catedraunesco/el-papel-delprofesorado-m-ngel-zabalzapresentation.

\section{PERFIL ACADÉMICO Y PROFESIONAL DE LAS AUTORAS}

Ruth Marlene Aguilar Feijoo. Doctora en Psicología Educativa, Magister en Administración Universitaria y Máster en Enseñanza y aprendizaje a Distancia por la UNED (España). Concluyendo la Tesis Doctoral en el Departamento de la Educación y Pedagogía Social de la UNED. Profesora Titular e investigadora en el Departamento de Ciencias de la Educación de la Universidad Técnica Particular de Loja-UTPL (Ecuador). Línea de investigación: Las competencias del docente universitario bimodal.

E-mail: raguilar@utpl.edu.ec

\section{DIRECCIÓN DE LAS AUTORA}

Universidad Técnica Particular de Loja (UTPL), San Cayetano Alto s/n, c/Marcelino Champagnat Dirección Postal 11-01-608, Loja-Ecuador

Ma José Bautista Cerro-Ruiz. Profesora Titular de la Universidad Nacional de Educación a Distancia (UNED). Doctora en Pedagogía, Licenciada en Ciencias Políticas y Sociología. Profesora del Departamento de Teoría de la Educación y Pedagogía Social en la UNED. Ha sido Secretaria Adjunta para el diseño de Títulos de Grado en la misma Facultad. Es experta en temas de Espacio Europeo de Educación Superior, Competencias y Educación Ambiental. Es miembro de la Cátedra UNESCO de Educación Ambiental y Desarrollo Sostenible de la UNED.

E-mail: mjbautistac@edu.uned.es

\section{DIRECCIÓN DE LAS AUTORAS}

Departamento de Teoría de la Educación y Pedagogía Social

Facultad de Educación.

UNED. c/ Juan del Rosal, 1428040 Madrid (España)

Fecha de recepción del artículo: 20/01/2015

Fecha de aceptación del artículo: 02/03/2015 


\section{Como citar este artículo:}

Aguilar Feijoo, R. M., y Bautista Cerro-Ruiz, M. J. (2015). Perfiles docentes y excelencia: un estudio en la Universidad Técnica Particular de Loja, Ecuador. RIED. Revista Iberoamericana de Educación a Distancia, 18 (2), 225-250. doi: http://dx.doi.org/10.5944/ried.18.2.13920. 